\title{
Development and Characteristics of Airstepping in Chronic Spinal Cats ${ }^{1}$
}

\author{
C. A. GIULIANI ${ }^{2}$ AND J. L. SMITH \\ Laboratory of Neuromotor Control, Department of Kinesiology, University of California, Los Angeles, California 90024
}

\begin{abstract}
Airstepping, walking-like movements of the hindlimbs, is a commonly observed behavior in chronic spinal animals when they are held vertically. The purpose of this study was to: describe the development of airstepping after spinalization and compare it to the onset of segmental reflexes, characterize the EMG pattern of muscle activity during spontaneous airstepping, and examine the effects of sensory perturbation on the characteristic pattern. Airstepping was analyzed during three conditions of tonic sensory perturbation which included: tail pinching, tape applied to one hindpaw, and immobilization of the ankle and knee in a plaster cast.

Seven adult cats were spinalized at $\mathrm{T}-12$, and bipolar electrode wires were surgically implanted in selected hindlimb muscles at the hip, knee, and ankle. Testing began within $48 \mathrm{hr}$ of transection. Segmental reflexes and pawshake responses were present in the first week; however, the earliest observed airstepping occurred during tail pinching at 2 weeks after surgery, and the average onset of spontaneous airstepping (without exteroceptive stimuli) was at 33 days. The average cycle period of spontaneous airstepping (691 msec) was comparable to the shortest periods reported for fictive rhythms and to treadmill walking between 1 and $2 \mathrm{~m} / \mathrm{sec}$. Intralimb coordination was characterized by flexor and extensor synergies typical of locomotion, while interlimb coordination was characterized by alternating cycles similar to that reported for treadmill walking and fictive locomotion. Neither intralimb nor interlimb patterns of coordination were altered by conditions of sensory perturbation, although cycle period and EMG recruitment level were variable.

Many characteristics of airstepping are similar to those of treadmill and fictive locomotion. Since airstepping is unencumbered by the effects of contact forces and belt speed present during treadmill locomotion and is uncomplicated by the effects of L-DOPA (needed to elicit fictive locomotion), it may provide a more reliable model with which to investigate the neural networks responsible for coordinating hindlimb locomotion.
\end{abstract}

Airstepping, walking-like movements of the hindlimbs without ground contact, is a common behavior in low-thoracic, cord-tran-

Received July 30, 1984; Revised November 2, 1984;

Accepted November 9, 1984

${ }^{1}$ This study was supported by National institutes of Health Grant NS 19864. We wish to acknowledge Nina Bradley, Gail Koshland, Dorothy Phillips, and Celia Sabin for their invaluable assistance in data collection.

2 To whom correspondence should be addressed. sected animals, including cats and dogs (Sherrington, 1910), guinea pigs (Brown, 1913), rabbits (Fayein and Viala, 1976), opossums (Hinsey and Cutting, 1936), and rats (Meisel and Rakerd, 1982). Although airstepping has not been reported in spinalized primates (Eidelberg et al., 1981) or human patients with verified cord transections (for review, see Eidelberg, 1981), a similar behavior is observed in human infants during early motor development (Theien and Fisher, 1982). Since the earliest reference by Freusberg (1874), who described the "mark-time" reflex, the behavior has been referred to as "spinal reflex stepping" (Sherrington, 1910), "stepping reflex" (Afelt, 1970), and recently as "walking behavior" (Thor el al., 1983). Despite the continued interest in airstepping, a detailed description of the neuromuscular patterns is not available.

The purpose of the present study was 3-fold. A description of the onset of airstepping after spinalization was our first objective. Since airstepping was reported by Sherrington (1910) as developing gradually and being more forceful in the "late spinal preparation," we compared the development of airstepping with the onset of segmental reflexes commonly tested to define the recovery process from spinal transection (Chambers et al., 1966). Our second purpose was to characterize both intralimb synergies and interlimb coordination of airstepping by describing activity patterns of selected extensor and flexor muscles at the hip, knee, and ankle joints in adult spinal cats with cord transections at T-12. Thirdly, we desired to determine the effects of tonic sensory perturbation, applied to a rcmotc locus or to a single limb, on the stability of the cycle characteristics and muscle recruitment patterns of airstepping.

We show that the characteristics of intralimb synergies and interlimb coordination are stable and very similar to those reported for treadmill walking in spinal cats (Forssberg et al., 1980a,b; Smith et al., 1982) and to fictive locomotion in the isolated-cord preparations (Baker et al., 1984; Grillner and Zangger, 1979). We conclude that airstepping is a model behavior with which to study the output of the central pattern generator for hindlimb locomotion (Grillner, 1981). Preliminary reports of our study have been published (Giuliani et al., 1982; Giuliani and Smith, 1983).

\section{Materials and Methods}

Surgery. Seven adult cats were spinalized under aseptic conditions using sodium pentobarbital (Nembutal, $35 \mathrm{mg} / \mathrm{kg}$ ) anesthesia administered intravenously. A partial laminectomy was performed at T-12, and the spinal cord was transected with microscissors. Completeness of the transection was assured when both ends of the severed cord retracted into the spinal canal and Gelfoam was packed between the rostral and caudal segments. Postoperative care and maintenance of spinal cats have been described previously (Smith et al., 1982).

Behavioral testing. Behavioral testing, initiated within $48 \mathrm{hr}$ after spinalization, was conducted 2 to 3 times a week for 6 weeks and weekly thereafter for 4 months. During each session, behaviors tested included tendon taps (deep tendon reflex), paw pinch to elicit flexion and crossed extension reflexes, and pin prick applied to the central pad of the hindpaw (plantar grasp reflex). Also, the cat's ability to support weight on the hindlimbs, pawshake responses (Sabin and Smith, 1984), and airstepping were assessed. 


\section{A. FIRST WEEK}

TA

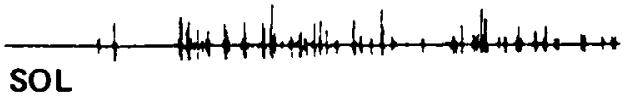

B. THIRD WEEK

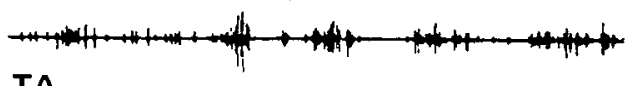

TA

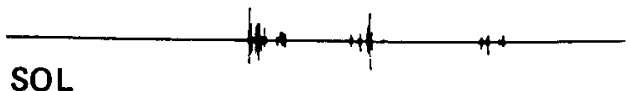

SOL

C. FIFTH WEEK
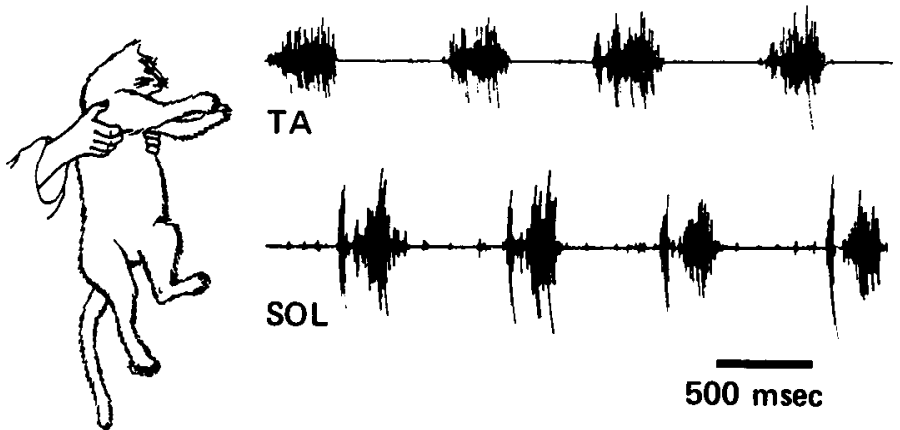

$500 \mathrm{msec}$

Figure 1. Development of the airstepping EMG pattern following spinalization. During the first week $(A)$, only low-level, tonic EMG activity was noted. By the third week $(B)$, two to three airstepping cycles were elicited immediately on lifting the cat, while sustained spontaneous airstepping was seen in the fitth week $(C)$.

To test for airstepping, the cat was supported under the axillae, lifted from a table and held vertically wilh the hindlimbs pendent (Fig. 1). Airstepping was recorded during four experimental conditions. In the first, we looked for airstepping to occur spontaneously, without any additional exteroceptive stimulation. In the second, tonic stimulation was provided by squeezing the cat's tail (tail pinching), a maneuver reported to facilitate airstepping (Sherrington, 1910). In the third, masking tape was wrapped around one hindpaw at the level of the central plantar pad. Tape was included as a stimulus condition because often airstepping that followed paw-shake responses appeared to have increased amplitudes and longer cycle periods. In the fourth condition, one hindlimb was casted to test the effects of perturbed segmental feedback. The ankle and knee were immobilized in the passivependent position at $110^{\circ}$ and $115^{\circ}$, respectively, in a plaster cast that was applied $t$ to $2 \mathrm{hr}$ prior to the recording session and removed immediately thereafter. Since sacral parasympathetic reflexes are reported to facilitate airstepping (Thor et al., 1983), the cat's bladder and bowel were emptied prior to each testing session.

Assessment. Airstepping was assessed primarily by EMG recordings. In all cats, bipolar fine-wire electrodes were implanted surgically in the soleus (SOL), lateral gastrocnemius (LG), tibialis anterior (TA), and vastus lateralis (VL) muscles of one hindlimb and the SOL of the contralateral limb using methods described by Smith et al. (1977). In three cats, the gluteus medius $(\mathrm{GM})$, iliopsoas $(\mathrm{IP})$, and anterior biceps femoris (ABF) muscles were also implanted. Prior to each recording session, the integrity of the electrodes was checked by stimulating each muscle through the implanted wires and observing or palpating the localized muscle contraction. Myopotentials were amplified $(x 1000)$, filtered with a high pass of $100 \mathrm{~Hz}$, and recorded on FM tape $\left(3^{3 / 4}\right.$ inches/sec) along with a binary code. Videotape synchronized to the FM tape by a binary code provided a record of the limb movements.

For each cat, 20 cycles of airstepping were analyzed for each of the four experimental conditions. Usually two series of 10 sequential steps were

chosen for computer analysis. The analogue signal for each channel was full-wave rectified, sampled at $5 \mathrm{kHz}$, and stored on disk as digital data Using established techniques (Sabin and Smith, 1984), the onset and termination of each burst were calculated as well as the burst duration. Also, the EMG was averaged with a 5-msec time constant, and the integrated value (I-EMG) for each burst was calculated.

In addition to the burst characteristics, the following parameters were determined and averaged for each record: cycle period, defined as the time interval between sequential SOL bursts of one hindlimb, and the onset latency, defined as the time interval between the beginning of the cycle and the onset of the muscle burst, normalized to the cycle period. Intralimb coordination was determined by the onset latencies of the flexor and extensor muscles, while interlimb coordination was defined by the onset latency of the contralateral SOL muscle.

\section{Results}

Onset of airstepping and initial recovery. The criterion used to determine the onset of reliable airstepping was the occurrence of 10 or more consecutive cycles in which movement, substantiated by EMG records, was observed at all three joints (hip, knee, and ankle). In all cats, airstepping was first observed only during tail pinching between 13 and 29 days after spinal transection, with an average of $19 \pm 6$ (SD) days. In contrast, the onset of spontaneous airstepping was observed between 28 and 39 days, with an average of $33 \pm 4$ days.

The development of airstepping was similar in all cats. Three days after spinal transection, the hindlimbs were flaccid and pendent when the cat was lifted to a vertical position. Tail pinching elicited brief episodes of small-amplitude, rhythmic movements in the hindlimbs, but no EMG pattern was evident in knee and ankle muscles implanted. Observation of the video records suggested that these movements were generated primarily at the hip, as segments below the knee and ankle appeared to remain flaccid. By the end of the first week, spontaneous movements of the hindlimbs were still absent, but epochs of tonic FMG activity were common, with occasional interruptions by alternating activity (Fig. 1A).

At 2 weeks, spontaneous hindlimb activity was minimal and consisted only of occasional kicking movements. At this time, airstepping was first observed during tail pinching and ceased immediately upon release of the tail. During the third postsurgical week, 2 to 4 bouts of cycling were frequently observed after a pawshake response, or immediately after lifting the cat to the vertical position. A typical response after lifting is shown in Figure 1B; in this record, two alternating cycles are shown interposed between tonic TA activity. By the fifth week, spontaneous airstepping was observed, and EMG patterns consisted of prolonged periods of alternating flexor and extensor activity (Fig. 1C).

Compared with other behaviors observed during the initial recovery period, the onset of spontaneous airstepping was late. A reflex response to tendon tap of the Achilles and quadriceps tendons was present immediately after spinalization and continued essentially unchanged. During the first week, paw pinch elicited a weak flexor withdrawal response that was limited to the ankle joint, and there was no response to pin prick of the central pad. The contralateral response to paw pinch included both crossed-extension and crossed-flexion responses, as evidenced by small amplitude ankle movement or by EMG activity without visible limb movement. The application of tape to the paw, with the cat held vertically, elicited a paw-shake response, while the contralateral limb hung flaccid. When the cat was placed in a quadrupedal standing position while supported under the thoracic area and by the tail, weight-supporting responses resulted in tonic ankle extensor EMG. There was, however, no observable weight support, as the hindlimbs collapsed immediately.

At 2 weeks, paw pinch elicited brisk flexion and crossed-extension responses that were often followed by a brief bout of cyclic movements resembling stepping. Toe curling was the first response to pin prick observed. The paw-shake response was easily elicited, and associated movements of the contralateral limb were observed. 
When the cat was placed in quadrupedal position, weight support was maintained momentarily with the hindlimbs in a flexed posture and the paws in a plantigrade position. Occasionally, one to two spontaneous steps were taken with the hindlimbs before balance was lost, and the cat's hindquarters fell over to one side.

By the third week, almost all testing and handling elicited short bouts of cyclic activity. Spontaneous airstepping, however, was not elicited when the cat was held vertically. Also at this time, periods of weight support increased, with the hindlimbs assuming more of an extended posture. From the fourth through the fifth week, all prior behaviors continued as described, and the only additional activity observed was the onset of spontaneous airstepping and longer periods of full weight support.

Characteristics of spontaneous airstepping. From the time of onset, the cycle period of spontaneous airstepping remained relatively constant for each cat. Among the seven animals tested, the average cycle period ranged from 513 to $992 \mathrm{msec}$, with an overall mean of $691 \mathrm{msec}$ (Table I). The cycle periods for both hindlimbs were usually equivalent. The shortest cycle period recorded was $387 \mathrm{msec}$, while the longest period was $1142 \mathrm{msec}$. Although there was a wide range in the duration of airstepping cycles between cats, the variability within cats was considerably less, as noted by the small average within-subject standard deviation (Table I).

Interlimb coordination of the hindlimbs was characterized by alternating stepping, with the hindlimbs approximately $180^{\circ}$ out-ofphase. That is, the onset of the contralateral SOL occurred on average at $49 \pm 6 \%$ of the cycle and ranged from 30 to $70 \%$ (Fig. 2). Intralimb coordination, expressed as the average onset latencies of the muscles within one hindlimb, is illustrated in Figure 3. Extensor and flexor muscle synergies were clearly evident in the airstepping pattern. Onsets of the LG and VL, when active, were tightly coupled to the onset of the ipsilateral SOL. The onsets of the flexors, TA and $\mathbb{P}$, were also tightly coupled, occurring at $72 \%$ of the cycle.

The mean SOL burst duration was $293 \mathrm{mscc}$ and occupied approximately $42 \%$ of the cycle period, whereas the mean burst duration of the TA was $159 \mathrm{msec}$ and occupied approximately $23 \%$ of the step cycle (Table I, Fig. 3). The duration of the extensor burst was correlated positively with cycle period, whereas the duration of the flexor burst was independent of the cycle period (Fig. 4, $A$ and $B$ ). Although the $\mathrm{SOL}, \mathrm{TA}$, and $\mathbb{P}$ were consistently active during spontaneous airstepping, the $V L$ and $L G$ were often silent or exhibited low-level activity, suggesting that the recruitment of these motor pools was minimal (Fig. 5A). The two hip extensors, GM and ABF, were not active during spontaneous airstepping; however, they did participate in the paw-shake response (Fig. 5D).

Tonic sensory perturbation. The effects of sensory stimulation and perturbation, produced by tail pinching, tape applied to the paw, and ankle-knee immobilization, were assessed by comparing patterns of muscle activity and cycle periods to those typical of spontaneous airstepping. The mean values for cycle period, SOL and TA burst durations, and normalized activity (I-EMG/burst duration) for all four conditions are given in Table I. A repeated-measures ANOVA was used to identify significant differences between condi-

TABLE I

Characteristics of spontaneous and perturbed airstepping

\begin{tabular}{|c|c|c|c|c|c|c|}
\hline \multirow[b]{2}{*}{ Conditions } & \multirow[b]{2}{*}{$n^{a}$} & \multirow{2}{*}{$\begin{array}{l}\text { Cycle Period } \\
\text { (msec) }\end{array}$} & \multicolumn{2}{|c|}{$\mathrm{SOL}$} & \multicolumn{2}{|c|}{ TA } \\
\hline & & & $\begin{array}{c}\mathrm{BD}^{b} \\
(\mathrm{msec})\end{array}$ & I-EMG/BD & $\begin{array}{c}\mathrm{BD} \\
\text { (misec) }\end{array}$ & I-EMG/BD \\
\hline Spontaneous & 7 & $691 \pm 60^{\circ}$ & $293 \pm 64$ & 0.07 & $159 \pm 37$ & 0.06 \\
\hline Tail pinch & 7 & $565 \pm 62$ & $273 \pm 60$ & 0.12 & $132 \pm 36$ & 0.06 \\
\hline Tape & 5 & $920 \pm 79^{d}$ & $376 \pm 81$ & 0.12 & $312 \pm 71^{d}$ & 0.03 \\
\hline Cast & 6 & $732 \pm 50$ & $415 \pm 33$ & 0.12 & $176 \pm 38$ & 0.10 \\
\hline
\end{tabular}

${ }^{a} n$, number of cats.

${ }^{b} \mathrm{BD}$, burst duration.

${ }^{c}$ Mean $\pm \mathrm{SD}$.

${ }^{\circ}$ Significant difference from spontaneous airstepping; $p<0.05$.

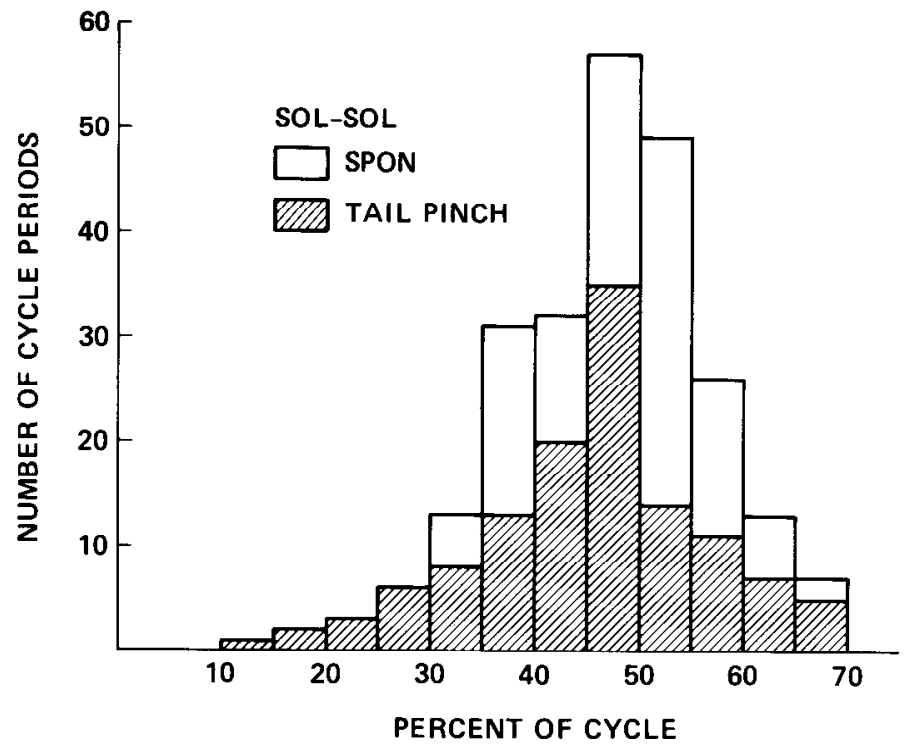

Figure 2. Interlimb coordination during airstepping. The temporal pattern of right and left ankle extensors ( $S O L-S O L)$, normalized to cycle period (percent of cycle), are plotted for 140 cycles (20/cat) under spontaneous and tail pinch conditions.

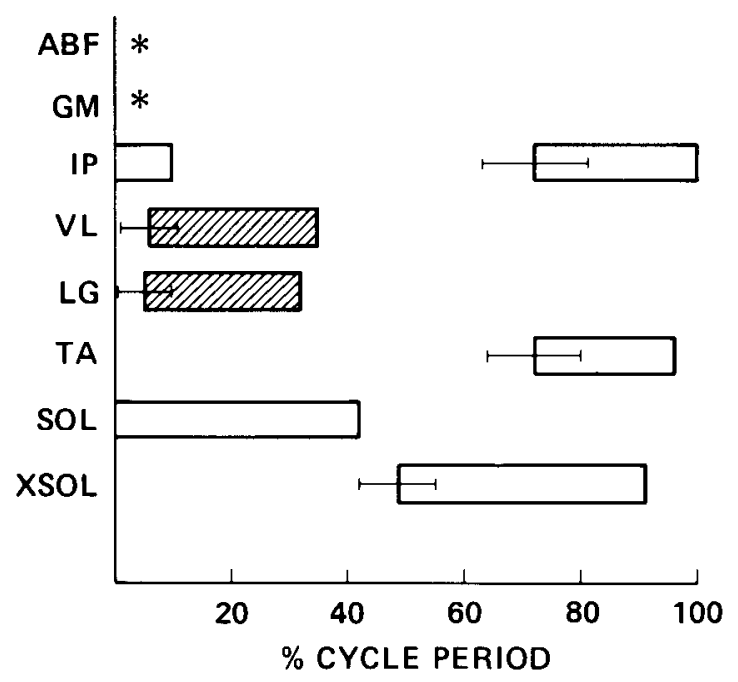

Figure 3. Muscle synergies for spontaneous airstepping. Typical intralimb patterns of selected flexor and extensor muscles are shown with the contralateral ankle extensor (XSOL). For each muscle, the mean burst duration and onset latency are normalized to the cycle period; horizontal bars show the average within-subject SD. As noted by the asterisk, the GM and ABF were not active during spontaneous airstepping, while the $V L$ and $L G$ showed variable recruitment, indicated by the striped bars (also see the text).

tions for each parameter, and post-hoc tests (Tukey's HSD) were performed when indicated.

Tail pinching had mixed effects on the cycle period, and the overall mean (565 msec) was not significantly different from that reported for spontaneous airstepping. Differences among cats, however, were instructive because a "floor effect" for facilitating the cycle period of alternating airstepping was suggested. The four cats with slower cycles (>700 msec) for spontaneous airstepping showed decreases that ranged trom 210 to $272 \mathrm{msec}$, while the three cats with faster spontaneous cycles ( $<600 \mathrm{msec}$ ) showed no change. Also, tail pinching occasionally resulted in stepping movements that were nonalternating, with the hindlimbs moving more in-phase, as indicated by a shift in the onset latency of the contralateral SOL to values less than $30 \%$ (Fig. 2). The cycle periods for these nonalternating steps, not averaged with the alternating steps, were very 


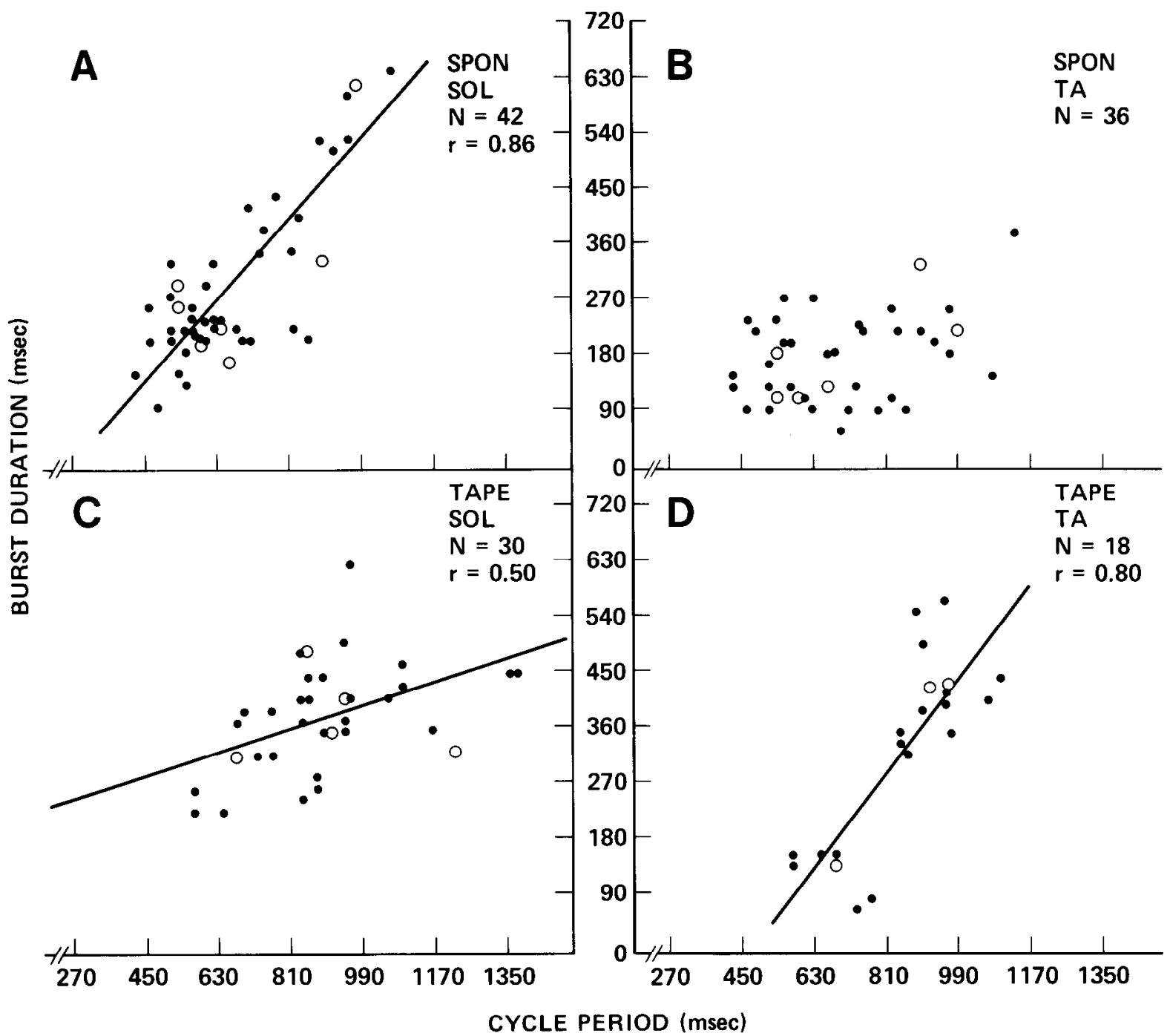

Figure 4. Burst duration of TA and SOL related to cycle period. Each dot (O) represents data for one cycle; six from each animal were selected to represent the range of cycle periods; the mean for each animal is shown by a circle (O). During spontaneous airstepping, SOL burst duration was correlated with cycle period $(A)$, while the TA burst duration was independent of the cycle period $(B)$. With tape applied to the hindpaw, both the SOL $(C)$ and TA burst durations $(D)$ were correlated with cycle period.

short ( $269 \pm 53 \mathrm{msec})$. Other behaviors observed periodically during tail pinching were micturition, increased flexion or extension position of the hip, and vigorous kicking movements.

From observations of video records, it was apparent that with tape on the paw, the ipsilateral hindlimb exhibited an increased range of motion and, in addition, the average cycle period was significantly longer. Also, the duration of TA activity increased significantly and was correlated with the cycle period (Fig. 4D). In contrast, the average duration of the SOL burst was not altered, and while the correlation between cycle period and SOL burst duration was still significant, the sensitivity, as measured by the slope, was reduced considerably (Fig. $4 \mathrm{C}$ ). In addition, the $\mathrm{LG}$ and the $\mathrm{VL}$ were active more often or tended to exhibit greater activity with tape on the paw (Fig. 5C); however, normalized activity levels for the SOL and TA were not affected (Table I). Another effect of the tape was an increase in the packaging within a single burst for both flexors and extensor muscles (Fig. $5 \mathrm{C}$ ). These subpackets of activity (10 to $12 \mathrm{~Hz}$ ) are typical of clonic oscillations reported in the spinal cat (Smith et al., 1982; Bradley and Smith, 1983).

Casting knee and ankle joints had no effect on either the cycle period, normalized activity, or the burst durations of the SOL or TA. The recruitment of those muscles (VL and LG) which exhibited variable and low-level EMG activity during spontaneous airstepping, however, was increased (Fig. $5 B$ ). Intralimb coordination, defined by onset latency of ankle muscles (SOL-TA), and the interlimb coordination (SOL-SOL) were essentially unchanged trom spontaneous airstepping across all conditions in which sensory input was altered (Fig. 6).

\section{Discussion}

Onset of airstepping. Following spinal transection, reflexes commonly tested to define the recovery process from spinal shock (Chambers et al., 1966; McCouch, 1947) were present within the first week. Airstepping, first observed during tail pinching in the second postoperative week, developed more slowly and by the fifth week (average of 33 days) was easily elicited by lifting the spinal cat to a vertical position (Fig. 1C). McCouch (1947) also reported that the average onset for stepping (trunk supported horizontally) was 31 days. Recently, Eidelberg et al. (1980) tested adult spinal cats for locomotion by supporting them above a treadmill and observed that stepping (no weight support) occurred between days 8 and 22 in five of six cats.

In contrast to the adult cat, kittens spinalized prior to 2 weeks of age exhibited airstepping within hours after transection (Bradley et al., 1983; Forssberg et al., 1980a). This age-dependent effect of spinal shock may be related to the small number of mature descend- 
A.
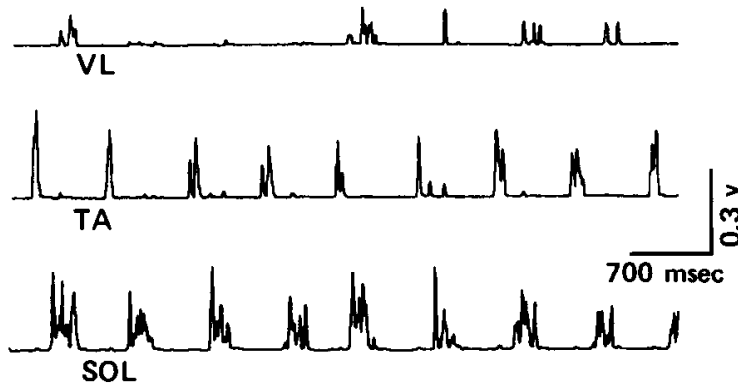

B.

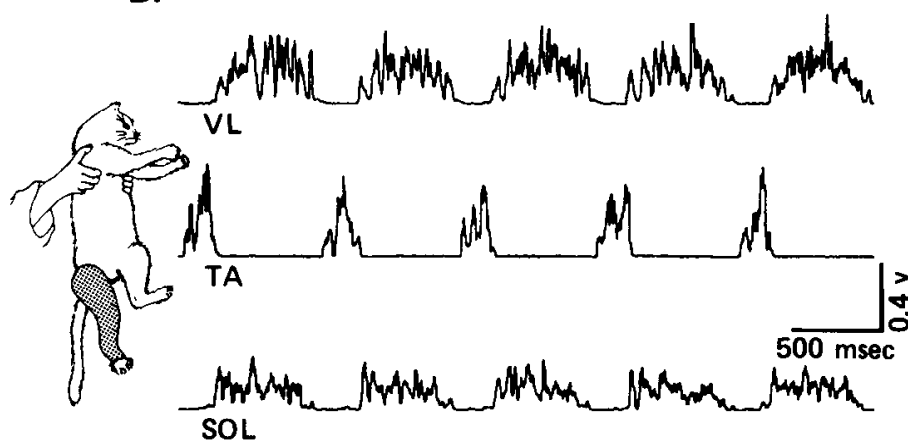

C.

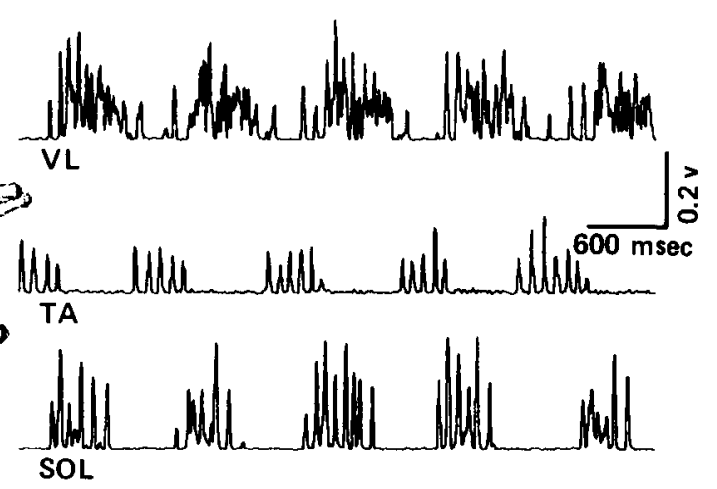

D.
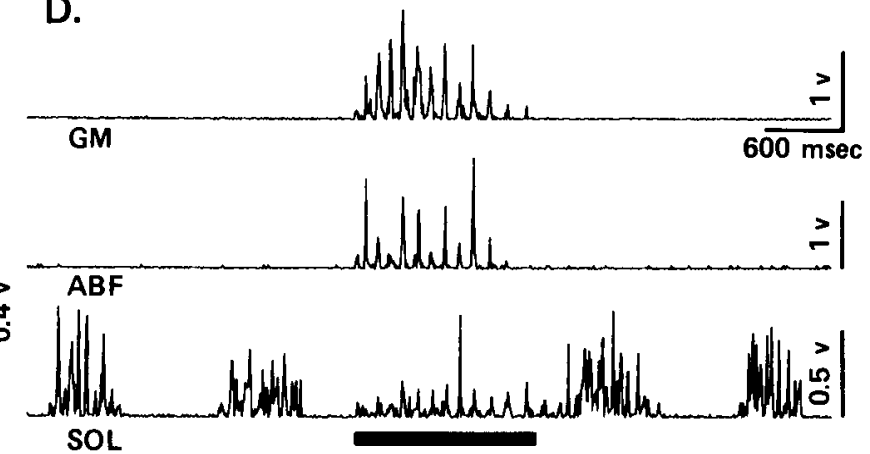

Figure 5. Intralimb recruitment patterns of airstepping during different conditions. During spontaneous airstepping (A), the SOL and TA are reciprocally

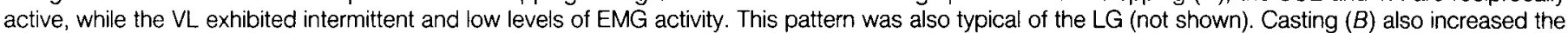

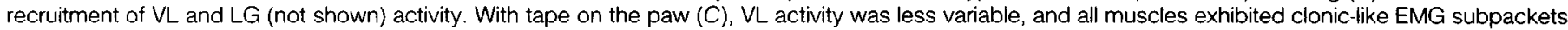

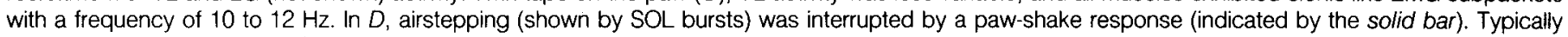
silent during airstepping, the GM and ABF participated during the paw-shake response.

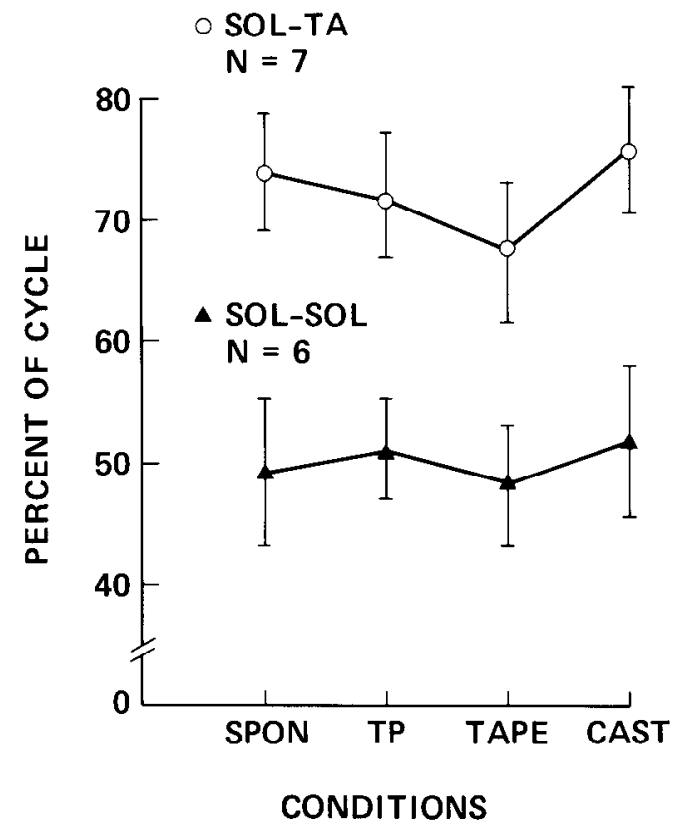

Figure 6. Stability of intralimb and interlimb coordination over testing conditions. Intralimb coordination, defined by the onset of flexor (TA) with respect to extensor (SOL) muscles for spontaneous airstepping (SPON), was unaffected by tail pinch (TP), tape, or timb immobilization in a cast. Interlimb coordination, defined by the temporal pattern of right and left ankle extensors (SOL-SOL), was out-of-phase and was not altered by the testing conditions. ing axons in the neonatal spinal cord that provide excitation (Liu and Chambers, 1980; Stelzner et al., 1975). Also, it is possible that during the postnatal weeks, rudimentary descending systems inhibit rather than facilitate patterned activity in the hindlimbs (Bradley and Smith, 1985; Robinson et al., 1983). Under these conditions, a spinal transection would disinhibit spinal circuits (also see Bekoff, 1981).

While it is believed that complex behaviors, such as airstepping, require more time to recover in the adult spinal animal (McCouch, 1947), the early onset ( $<48 \mathrm{hr}$ ) of the paw-shake response (Sabin and Smith, 1984) is contrary to this belief. It may be that the initiation of the paw-shake response is intrinsic to the lumbosacral cord and not dependent on supraspinal facilitation. In contrast, the delay of airstepping may be related to the lack of tonic input from supraspinal centers normally required to facilitate locomotion. In the acute spinal cat paralyzed with curare, administration of a noradrenergic precursor (L-DOPA), which is thought to mimic tonic facilitation provided by descending noradrenergic fibers, may elicit fictive locomotion, but tonic stimulation of remote dorsal roots and dorsal column fibers is often required to initiate rhythmic activity (Grillner and Zangger, 1979). Airstepping was also facilitated by tonic input from remote loci, including tail pinching (Meisel and Rakerd, 1982), bladder distension (Thor et al., 1983), and perineum stimulation (Sherrington, 1910). Later in recovery, however, initiation of airstepping gradually becomes less dependent on tonic input from remote loci and more responsive to afferent input from the hindlimbs. Thus, it is possible that some changes intrinsic to the lumbosacral cord occur over time to compensate for the lack of supraspinal facilitation.

It appears that a substantial amount of reorganization of limb afferents occurs during the first month of recovery in segments caudal to the spinal lesion (for review, see Mendell, 1984). Increased afferent terminals within the dorsal horn (Murray and Goldberger, 
TREADMILL SPEED $(\mathrm{m} / \mathrm{sec})$

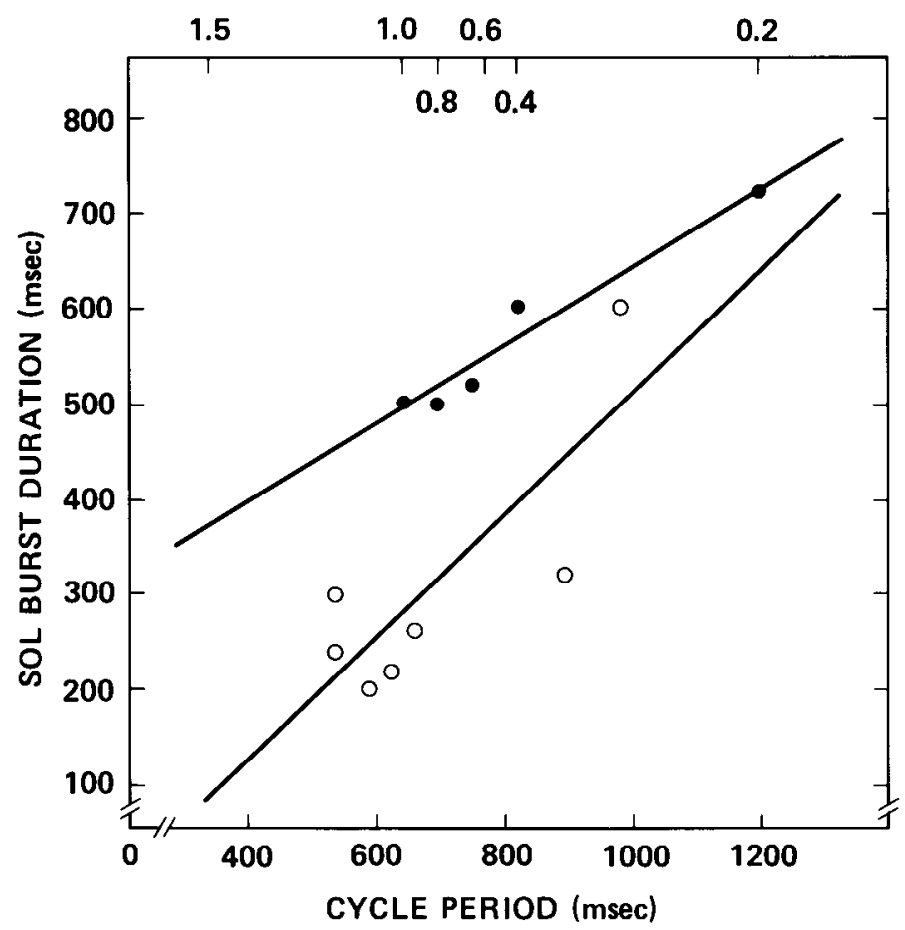

Figure 7. Cycle period for airstepping and treadmill locomotion related to SOL burst duration. For the lower regression line $(r=0.86 ; p<0.05)$, mean SOL burst duration was plotted against mean airstep cycle for 7 cats. For the upper regression line $(r=0.9 ; p<0.05)$, unpublished data from one spinal cat with good weight-support on the treadmill was used to plot the mean SOL burst duration for 20 steps against the cycle period for treadmill locomotion at five speeds. Treadmill speeds are indicated on the upper scale of the abscissa.

1974), expanded receptive fields of cutaneous afferents (Brenowitz and Pubols, 1981), and increased projection frequencics of la afferents to homonymous (Nelson and Mendell, 1979) and to heteronomous motoneurons (Mendell et al., 1982) provide evidence of anatomical and physiological changes following spinal cord transection. Although the changes responsible for the gradual onset of airstepping are unknown, it is possible that augmented afferent input from the limb is able to activate the central pattern generator itself, the output cells of the generator, or both (Chandler and Goidberg 1982; Kawahara and Mori, 1982)

Locomotor patterns: Airstepping, fictive, treadmill. Many characteristics of airstepping are similar to those of fictive and treadmill locomotion. The cycle periods of airstepping are comparable to the shortest period lengths ( 0.6 to $1.0 \mathrm{sec}$ ) reported for fictive rhythms (Baker et al., 1984; Grillner and Zangger, 1979). In these isolated cord preparations, cycle periods are generally long ( 1.5 to $3.0 \mathrm{sec})$; however, stimulation of dorsal roots or dorsal column fibers may decrease the cycle period (Grillner and Zangger, 1979). Likewise, tail pinching decreased the average airstep cycle period in the cats that had slower spontaneous cycles (>600 msec)

The frequency of airstepping was comparable to that of treadmill walking between 1 to $2 \mathrm{~m} / \mathrm{sec}$, in the spinal cat (Fig. 7). Spinal cats, however, are not usually capable of good weight-supporting locomotion at speeds greater than $1.0 \mathrm{~m} / \mathrm{sec}$ (Forssberg et al., 1980a,b; Giuliani et al., 1984; Smith et al., 1982), which suggests that while postural constraints for locomotion may not be satisfied, the generation of rhythmic activity is not impaired.

Interlimb coordination between the hindlimbs in airstepping and in fictive locomotion (Grillner and Zangger, 1979; Baker et al., 1984) is typically alternating. However, with increased stimulation during fictive locomotion and occasionally during airstepping with tail pinch- ing, a nonalternating hindlimb pattern more typical of a gallop was elicited. Coupling between the two hindlimbs seems particularly strong, and although one hindlimb can airstep by itself or step aione on the treadmill when the contralateral paw is restrained (Grillner and Rossignol, 1978), it is difficult to uncouple the interlimb coordination if both limbs are allowed to participate. Thus, when tape was applied to one paw during airstepping, the effect of a lengthened cycle was reflected in the contralateral limb. Only with limb deafferentation was true uncoupling of the hindlimbs observed during airstepping (Giuliani and Smith, 1983).

Intralimb coordination is similar for airstepping, fictive locomotion (Grillner and Zangger, 1979), and treadmill walking (Halbertsma, 1983). In all three, an extensor synergy is reciprocally active with a flexor synergy at the hip, knee, and ankle. Also, the duration of extensor activity is correlated with the cycle period, while the flexor activity is not. At any cycle period, however, the predicted length of extensor activity during airstepping is 100 to $120 \mathrm{msec}$ shorter than that for treadmill walking (Fig. 7). This observation is consistent with the findings of Duysens and Pearson (1980), who showed that the duration of ankle extensor activity is related to the contractile tension developed during stance. In the pendent limb, contractile tensions are expected to be low and to decay quickly, and as a consequence, the extensor burst would be terminated early, whereas, with casting at the anklc joint, contractile tensions may have increased due to reflex activity produced by force feedback and the burst durations of the ankle extensors may have increased an average of $122 \mathrm{msec}$, while the average duration of the flexor burst (TA) was not affected (Table I).

Recruitment of extensor muscles during airstepping also mimicked patterns reported for treadmill walking. Fast-contracting extensors, such as the LG, VL, GM, and ABF, are either silent or participate minimally during airstepping and treadmill walking at speeds less than $1.0 \mathrm{~m} / \mathrm{sec}$ (Carter and Smith, 1984; Giuliani et al., 1984; Smith et al., 1977; Walmsley et al., 1978). During airstepping, however, recruitment levels of the $L G$ and VL were increased during tape application and casting. With tape as well as the cast, cutaneous input from the paw, known to selectively recruit fast-contracting muscle units in ankle extensors (Pinter et al., 1982), may have interacted directly or indirectly with the output of the central pattern generator to increase activity levels of fast extensors.

Airstepping: A model behavior. Airstepping appears to be a good model with which to study the output of the lumbosacral neural networks for locomotion in spinal cats. Many characteristics of airstepping are similar to those of treadmill locomotion, as well as fictive locomotion. Since the neuromuscular patterns of treadmill locomotion are influenced by the contact forces and the speed of the belt (Duysens and Pearson, 1980; Grillner and Rossignol, 1978), the patterns produced during airstepping may prove to be a better representation of the "natural" output of the spinal generator. Although the basic locomotor pattern may be revealed with the acute isolated-cord preparation (Baker et al., 1984; Grillner and Zangger, 1979), the preparation is not very reliable, and the effects of L-DOPA (needed to initiate fictive activity) on the central pattern generator are poorly understood (see Grillner, 1981, for a review). Thus, airstepping may provide an excellent behavior with which to investigate the intralimb and interlimb control mechanisms of the neural networks responsible for coordinating hindlimb locomotion.

\section{References}

Afelt, Z. (1970) Reflex activity in chronic spinal cats. Acta Neurobiol. Exp. (Warsz.) 30: 129-144.

Baker, L. L., S. H. Chandler, and L. J. Goldberg (1984) L-Dopa-induced locomotor-like activity observed in ankle flexor and extensor muscle nerves of chronic and acute spinal cats. Exp. Neurol. 86: 515-526.

Bekoff, A. (1981) Embryonic development of the neural circuitry underlying motor coordination. In Studies in Developmental Neurobiology, W. M. Cowan, ed., pp. 134-170, Oxford University Press, New York.

Bradley, N. S., and J. L. Smith (1983) Neuromuscular oscillations about the ankle joint in the chronic spinalized cat. Soc. Neurosci. Abstr. 9: 526. 
Bradley, N. S., and J. L. Smith (1985) Early onset of hindlimb paw-shake responses in spinal kittens: New perspective in motor development. Dev. Brain Res. 17: 301-303.

Bradley, N. S., J. L. Smith, and J. R. Villablanca (1983) Absence of hindlimb tactile placing in spinal cats and kittens. Exp. Neurol. 82: 73-88.

Brenowitz, G. L., and L. M. Pubols (1981) Increased response to sural nerve input in the dorsal horn following chronic spinal cord hemisection. Brain Res. 208: 421-425.

Brown, T. G. (1913) The phenomenon of "narcosis progression" in mammals. Proc. R. Soc. Lond. [Biol.] 86: 140-164.

Carter, M. C., and J. L. Smith (1984) Blending of intralimb synergies during the coordination of two distinct rhythmical movements. Soc. Neurosci. Abstr. 10: 631.

Chambers, W. W., C. N. Liu, G. P. McCouch, and E. D'Aquili (1966) Descending tracts and spinal shock in the cat. Brain 89: 337-390.

Chandler, S. H., and L. J. Goldberg (1982) Intracellular analysis of synaptic mechanisms controlling spontaneous and cortically induced rhythmical jaw movements in the guinea pig. J. Neurophysiol. 48: 126-138.

Duysens, J., and K. G. Pearson (1980) Inhibition of flexor burst generated by loading ankle extensor muscles in walking cats. Brain Res. 187: $321-$ 332.

Eidelberg, E. (1981) Consequences of spinal cord lesions upon motor function, with special reference to locomotor activity. Prog. Neurobiol. 17: 185-202.

Eidelberg. E., J. L. Story, B. L. Meyer, and J. Nystel (1980) Stepping by chronic spinal cats. Exp. Brain Res. 40: 241-246.

Eidelberg, E., J. G. Walden, and L. H. Nguyen (1981) Locomotor control in macaque monkey. Brain 104: 647-663.

Fayein, N. A., and D. Viala (1976) Development of locomotor activities in young chronic spinal rabbits. Neurosci. Lett. 3: 329-333.

Forssberg, H., S. Grillner, and J. Halbertsma (1980a) The locomotion of the low spinal cat. I. Coordination within a hindlimb. Acta Physiol. Scand. 108: 269-281

Forssberg, H., S. Grillner, and J. Halbertsma (1980b) Locomotion of the low spinal cat. II. Interlimb coordination. Acta Physiol. Scand. 108: 283295.

Freusberg, A. (1874) Reflexbewegungen beim hunde. Pflugers Arch. 9: 358391.

Giuliani, C. A., M. C. Carter, and J. L. Smith (1984) Return of weightsupported locomotion in the adult spinal cat. Soc. Neurosci. Abstr. 10: 632.

Giuliani, C. A., C. Sabin, and J. L. Smith (1982) Airstepping in the chronic spinal cat. Soc. Neurosci. Abstr. 8: 164.

Giuliani, C. A., and J. L. Smith (1983) Onset and perturbation of airstepping in the chronic spinalized cat. Soc. Neurosci. Abstr. 9: 358.

Grillner, S. (1981) Control of locomotion in bipeds, tetrapods, and fish. in Handbook of Physiology. Section 1: The Nervous System, Vol. II, Part 2: Motor Control, V. B. Brooks, ed., pp. 1179-1236, Williams \& Wilkins, Baltimore.

Grillner, S., and S. Rossignol (1978) On the initiation of the swing phase of locomotion in chronic spinal cats. Brain Res. 146: 269-277.

Grillner, S., and P. Zangger (1979) On the central generation of locomotion in the spinal cat. Exp. Brain Res. 34: 241-261.
Halbertsma, J. (1983) The stride cycle of the cat: the modelling of locomotion by computerized analysis of automatic recordings. Acta Physiol. Scand. Suppl. 521: 1-75.

Hinsey, J. C., and C. C. Cutting (1936) Reflexes in the spinal opposum. J. Comp. Neurol. 64: 375-388.

Kawahara, K., and S. Mori (1982) A two-compartment model of the stepping generator: Analysis of the roles of a stage-setter and a rhythm generator. Biol. Cybern. 43: 225-230.

Liu, C. N., and W. W. Chambers (1980) Collateral sprouting in the spinal cord. In The Spinal Cord and its Reaction to Traumatic Injury, W. F. Windle, ed., pp. 219-236, Marcel Dekker, New York.

McCouch, G. P. (1947) Reflex development in the chronically spinal cat and dog. J. Neurophysiol. 10: 425-428.

Meisel, R. L., and B. Rakerd (1982) Induction of hindlimb stepping movements in rats spinally transected as adults or as neonates. Brain Res. 240 : 353-356.

Mendell, L. M. (1984) Modifiability of spinal synapses. Physiol. Rev. 64: 260324.

Mendell, L. M., T. C. Cope, and S. G. Nelson (1982) Plasticity of the group la fiber pathway to motoneurons. In Changing Concepts of the Nervous System, A. Morrison and P. Strick, eds., pp. 69-78, Academic Press, Inc., New York.

Murray, M., and M. E. Goldberger (1974) Restitution of function and collateral sprouting in the cat spinal cord: The partially hemisected animal. J. Comp. Neurol. 158: 19-36.

Neison, S. G., and L. M. Mendell (1979) Enhancement in la-motoneuron synaptic transmission caudal to chronic spinal cord transection. J. Neurophysiol. 42: 642-654.

Pinter, M., R. E. Burke, M. J. O'Donovan, and R. P. Dum (1982) Supraspinal facilitation of cutaneous polysynaptic EPSPs in cat medial gastrocnemius motoneurons. Exp. Brain Res. 45: 133-143.

Robinson, G. A., C. T. Leonard, and M. E. Goldberger (1983) Development of placing reactions in spinal cats. Soc. Neurosci. Abstr. 9: 60 .

Sabin, C., and J. L. Smith (1984) Recovery and perturbation of paw-shake responses in spinal cats. J. Neurophysiol. 51: 680-688

Sherrington, C. S. (1910) Flexion-reflex of the limb, crossed extension-reflex stepping and standings. J. Physiol. (Lond.) 40: 28-121.

Smith, J. L., L. A. Smith, R. F. Zernicke, and M. Hoy (1982) Locomotion in exercised and nonexercised cats cordotomized at two or twelve weeks of age. Exp. Neurol. 76: 393-413.

Smith, J. L., V. R. Edgerton, B. Betts, and T. Collatos (1977) EMG of slow and fast ankle extensors of cat during posture, locomotion, and jumping. J. Neurophysiol. 40: 503-513.

Stelzner, D. J., W. B. Ershler, and E. D. Weber (1975) Effects of spinal transection in neonatal and weanling rats: Survival of function. Exp. Neurol. 46: $156-177$.

Theien, E. and D. M. Fisher (1982) Nowborn stepping: An explanation for a "disappearing" reflex. Dev. Psychol. 18: 760-775.

Thor, K. B., J. R. Roppolo, and W. C. deGroat (1983) Naloxone-induced micturition in unanesthetized paraplegic cats. J. Urol. 129: 202-205.

Walmsley, B., J. A. Hodgson, and R. E. Burke (1978) Forces produced by medial gastrocnemius and soleus muscles during locomotion in freely moving cats. J. Neurophysiol. 41: 1203-1216. 